\begin{tabular}{|c|c|}
\hline Title & Preparation and optical property of gallium zinc oxynitride powder and nanocrystals with sawtooth-like appearance \\
\hline Author(s) & Kamura, A tsuo; Masubuchi, Y uji; Motohashi, Teruki; Kikkawa, Shinichi \\
\hline Citation & $\begin{array}{l}\text { Material s research bulletin, } 87,130-134 \\
\text { https://doi.org/10.1016/.materresbull.2016.11.013 }\end{array}$ \\
\hline Issue Date & 2017-03 \\
\hline Doc URL & http:/hdl.handle.net/2115/72722 \\
\hline Rights & $\begin{array}{l}\text { (c) 2017, Elsevier. Licensed under the Creative Commons A ttribution-NonCommercial- NoDerivatives 4.0 International } \\
\text { http://creativecommons.org/icenses/by-nc-nd/4.0/ }\end{array}$ \\
\hline Rights(URL) & http://creativecommons.org/icenses/by-nc-nd/4.0/ \\
\hline Type & article (author version) \\
\hline File Information & MRB-87-2017-130-134-Masubuchi.pdf \\
\hline
\end{tabular}

Instructions for use 


\section{Preparation and optical property of gallium zinc oxynitride powder and nanocrystals with sawtooth-like appearance}

Atsuo Kamura ${ }^{1}$, Yuji Masubuchi*, Teruki Motohashi ${ }^{2}$, and Shinichi Kikkawa

Affiliation: Faculty of Engineering, Hokkaido University, N13 W8, Kita-ku, Sapporo, 060-8628, Japan

${ }^{*}$ Corresponding author: Y. Masubuchi; Address: Faculty of Engineering, Hokkaido University, N13 W8, Sapporo, 060-8628, Japan; Tel: +81-(0)11-706-6742, $\quad$ Fax: $\quad+81-(0) 11-706-6740 ; \quad$ E-mail: yuji-mas@eng.hokudai.ac.jp

Present addresses:

${ }^{1}$ Department of Applied Chemistry, Graduate School of Advanced Science and Engineering, Waseda University, Ohkubo 3-4-1, Shinjuku-ku, Tokyo, 169-8555, Japan

${ }^{2}$ Department of Materials and Life Chemistry, Kanagawa University, 3-27-1 Rokkakubashi, Kanagawa-ku Yokohama, 221-8686, Japan 


\section{Abstract:}

Gallium zinc oxynitride powder and its nanocrystals were obtained by nitridation of a $\mathrm{ZnGa}_{2} \mathrm{O}_{4}$ precursor under $\mathrm{NH}_{3}$ flow. The nitrided powder had the chemical composition of $\left(\mathrm{Ga}_{0.75} \mathrm{Zn}_{0.23} \square_{0.02}\right)\left(\mathrm{N}_{0.71} \mathrm{O}_{0.29}\right)$, where $\square$ indicates a vacancy, and showed improved crystallinity with a decrease in cationic vacancies compared to that for pristine gallium oxynitride without zinc. Gallium zinc oxynitride nanocrystals with rod-like morphology were obtained by nitridation of the oxide precursor on a $\mathrm{NiO}$ pellet. The nanorod crystals were several hundred nanometers wide and several micrometers long. The nanorods had a sawtooth appearance with triangular grains aligned along the length direction of the rods. The nanocrystals with a chemical composition of $\mathrm{Ga} / \mathrm{Zn}=$ 0.80/0.20 exhibited a broad cathodoluminescence emission at $2.1 \mathrm{eV}$, similar to the powder products.

KEYWORDS: A. nanostructures, A. nitrides, B. crystal growth, B. optical properties, D. defects 


\section{Introduction}

Gallium nitride with nanostructures, such as nanowires, nanorods, and plate-like crystals, has been widely studied for various applications including nanoscale UV lasers, high-speed field effect transistors, and electron emitters [1-6]. Their optical and electronic properties of gallium nitride are strongly dependent on the chemical composition and the morphology. A large number of investigations on the doping of GaN have been reported for many different dopant elements, such as Zn, Mg, Al, C, and Si [7,8]. Lattice defects, such as gallium interstitials, gallium site vacancies, and oxygen impurities also affect the optical and electronic properties due to the formation of additional electronic levels into the forbidden band [9-11].

Gallium oxynitride (GaNO), consisting of both oxide and nitride ions as its main anion component, has been prepared by nitridation of amorphous gallium oxide in an ammonia flow [12]. The GaNO adopting a wurtzite-like structure, has the chemical composition $\left(\mathrm{Ga}_{0.89 \square 0.11}\right)\left(\mathrm{N}_{0.67} \mathrm{O}_{0.33}\right)$, where $\square$ indicates a gallium site vacancy caused by $\mathrm{N}^{3-}$ substitution with $\mathrm{O}^{2-}$. Nanowires of GaNO have been obtained by ammonolysis of an amorphous gallium oxide powder containing a few percent of $\mathrm{Ni}$ or Co additives [13]. Scanning 
transmission electron microscopy (STEM) observation of GaNO nanowires indicates that the growth direction is parallel to the hexagonal $c$-plane, and there is a stacking disorder of several atomic layers between biphasic wurtzite and zinc-blende lattices along the hexagonal c-axis [14]. Cathodoluminescence (CL) spectra of GaNO nanowires revealed a broad emission and persistent photoconductivity (PPC) under UV irradiation [15]. Both the broad CL emission and PPC are considered to be induced by gallium vacancies caused by the oxide ion substitution of nitride ions in hexagonal GaN.

$\mathrm{ZnO}$ doping of $\mathrm{GaN}$ forms wurtzite-type $\mathrm{Ga}_{1-x} \mathrm{Zn}_{x} \mathrm{~N}_{1-x} \mathrm{O}_{x}(\mathrm{GaZnNO})$ solid solutions and has been reported to improve the crystallinity by decreasing the number of gallium vacancies [16]. However a formation of cation vacancy and its relationship to the optical property have not investigated in the literature. The oxynitride solid solutions have been widely studied for an application as a visible light-driven photocatalyst for the overall water splitting reaction [17-20]. GaZnNO has been prepared by several preparation methods; ammonolysis of $\mathrm{Ga}_{2} \mathrm{O}_{3}$ and $\mathrm{ZnO}$ mixtures, crystalline $\mathrm{ZnGa}_{2} \mathrm{O}_{4}$, and $\mathrm{Ga}-\mathrm{Zn}$ layered double hydroxides, and nitridation using urea as a nitrogen source [21-23]. Cationic vacancies have been neglected in discussions on the optical and catalytic properties of GaZnNO. 
To the best of our knowledge, there have been only a few reports on the nanostructural control of the GaZnNO [24,25]. Nanowires of the $\mathrm{Ga}_{1-x} \mathrm{Zn}_{x} \mathrm{~N}_{1-x} \mathrm{O}_{x}$ solid solution have been synthesized with a relatively low $\mathrm{Zn}$ content of less than $\mathrm{x}=0.12$, and their electronic properties were reported in relation to the $\mathrm{Zn}$ contents [24].

Control of the growth of GaZnNO nanocrystals may provide a possibility to improve the properties, such as $\mathrm{CL}$ emission, catalytic activity and electronic conductivity due to improved crystallinity. In the present study, GaZnNO powder and nanocrystals were obtained by an ammonolysis reaction of $\mathrm{ZnGa}_{2} \mathrm{O}_{4}$ without $\mathrm{NiO}$ and on a $\mathrm{NiO}$ compact as a catalyst, respectively. The morphology, growth mechanism and optical properties of the GaZnNO nanocrystals were investigated, and the CL emissions of both powder and nanocrystals are discussed in relation to gallium site vacancies.

\section{Experimental procedure}

$\mathrm{ZnGa}_{2} \mathrm{O}_{4}$ was prepared via the solid-state reaction of $\mathrm{Ga}_{2} \mathrm{O}_{3}(99.9 \%$, Kojyundo Chem. Lab. Co.) and ZnO (99\%, Wako Pure Chemicals Co.). These powders were mixed in a stoichiometric composition with ethanol in an agate 
mortar. The mixed powder was calcined at $1200^{\circ} \mathrm{C}$ for $12 \mathrm{~h}$ in air. The obtained $\mathrm{ZnGa}_{2} \mathrm{O}_{4}$ was nitrided at $750{ }^{\circ} \mathrm{C}$ for $10 \mathrm{~h}$ in an ammonia flow of $50 \mathrm{~mL} / \mathrm{min}$ to form a GaZnNO powder. Such high flow rate of ammonia was necessary to nitride the oxide precursor before thermal decomposition of ammonia to nitrogen and hydrogen as reported in a formation of oxynitrides by ammonolysis reaction [26]. The growth of GaZnNO nanocrystals was studied by a similar nitridation of $\mathrm{ZnGa}_{2} \mathrm{O}_{4}$ on a $10 \mathrm{~mm}$ diameter and $3 \mathrm{~mm}$ thick $\mathrm{NiO}$ catalyst pellet. As a reference, GaNO powders and nanowires were also obtained from $\mathrm{Ga}_{2} \mathrm{O}_{3}$ precursor under the same nitridation conditions as that for $\mathrm{ZnGa}_{2} \mathrm{O}_{4}$.

X-ray diffraction (XRD) patterns were collected using a diffractometer with $\mathrm{Cu} \mathrm{K}_{\alpha}$ radiation (Ultima IV, Rigaku). The chemical compositions of $\mathrm{Ga}$ and Zn were measured using X-ray fluorescence spectroscopy (XRF; SEA6000VX-SII, Hitachi). The oxygen and nitrogen contents were determined with an oxygen/nitrogen analyzer (EMGA-620W, Horiba) using $\mathrm{Y}_{2} \mathrm{O}_{3}$ and $\mathrm{Si}_{3} \mathrm{~N}_{4}$ as respective references. The microstructure of product samples placed on carbon tape was observed using scanning electron microscopy (SEM; JSM-6390LV, Jeol) and field emission SEM (FE-SEM; JSM-6500F, Jeol). Transmission electron microscopy (TEM) samples were prepared by focused ion 
beam milling (FIB; JIB-4600F, Jeol) and supported on micro-grid coated Mo mesh. TEM and STEM observations were performed using microscopes, JEM-2010 (Jeol) and HD-2000 (Hitachi), respectively. Elemental analysis was also performed using energy dispersive X-ray spectroscopy (EDX). CL spectra of the nitrided products were measured at room temperature using FE-SEM at an accelerating voltage of $15 \mathrm{kV}$ attached to a CL system (SU6600CL, Hitachi).

\section{Results and discussion}

\subsection{Gallium zinc oxynitride powder}

Fig. 1 shows XRD patterns of the nitrided powder products obtained from $\mathrm{ZnGa}_{2} \mathrm{O}_{4}$ and $\mathrm{Ga}_{2} \mathrm{O}_{3}$ without the $\mathrm{NiO}$ catalyst pellet. The XRD pattern of the GaNO powder is slightly different from the diffraction pattern calculated for the wurtzite-type GaN lattice [27] shown at the bottom. Biphasic wurtzite and zinc-blende structures have been reported for GaNO powders and nanowires, and may be related to a stacking disorder along the $c$-axis of the hexagonal wurtzite lattice. Such disorder was directly observed on GaNO nanowires using high resolution STEM [14]. The biphasic nature decreased in the GaZnNO powder obtained by nitridation of the $\mathrm{ZnGa}_{2} \mathrm{O}_{4}$ precursor and the $\mathrm{XRD}$ intensity 
ratio was similar to that of hexagonal wurtzite type GaN. The stacking disorder was thus decreased by the $\mathrm{Zn}$ doping into the GaNO. The narrower diffraction lines suggest improved crystallinity in the GaZnNO powder. The lattice parameters were estimated assuming the hexagonal wurtzite type structure to be $a=0.3187(2) \mathrm{nm}$ and $c=0.5181(4) \mathrm{nm}$ for GaNO, which increased to $a=$ $0.3204(1) \mathrm{nm}$ and $c=0.5195(1) \mathrm{nm}$ for GaZnNO, because $\mathrm{Zn}^{2+}$ has a larger ionic radius than $\mathrm{Ga}^{3+}[29]$. Their chemical compositions were estimated from both oxygen/nitrogen analysis and XRF measurements. While GaNO powder contains approximately 8 at $\%$ of cation vacancies (Table 1 ), the $\mathrm{Zn}$ doping of GaZnNO significantly decreased the cation vacancy to 2 at $\%$. The Zn/Ga ratio in GaZnNO decreased from that in the $\mathrm{ZnGa}_{2} \mathrm{O}_{4}$ precursor because $\mathrm{Zn}$ is more volatile than $\mathrm{Ga}$ under a strong reductive atmosphere.

\subsection{Gallium zinc oxynitride nanocrystals}

Nanocrystal growth was investigated by the nitridation of $\mathrm{Ga}_{2} \mathrm{O}_{3}$ and $\mathrm{ZnGa}_{2} \mathrm{O}_{4}$ on $\mathrm{NiO}$ catalyst pellets. Nitrided products obtained on the $\mathrm{NiO}$ pellets were observed using SEM, and the results are shown in Fig. 2. Several nanowires with widths of 20 to $100 \mathrm{~nm}$ and ca. $20 \mu \mathrm{m}$ long were obtained with 
GaNO grains as the nitridation product from $\mathrm{Ga}_{2} \mathrm{O}_{3}$. The nanowires have smooth surfaces and the morphology was similar to that previously reported for nanowire prepared by nitridation of an amorphous Ga-Ni oxide [13, 14]. The previous nanowires grown from the amorphous precursor contained a large amount of cation vacancies and a stacking disorder [14]. The number of nanocrystals in the nitrided product increased for preparation from the $\mathrm{ZnGa}_{2} \mathrm{O}_{4}$ precursor on a NiO pellet, as shown in Fig. 2(b). The morphology changed from that for a plain nanowire to the characteristic nanorods with widths above 200 $\mathrm{nm}$ and $5 \mu \mathrm{m}$ long. The magnified image in Fig. 2(c) shows triangular grains that are aligned along the rod and have a sawtooth-like appearance. The $\mathrm{Zn} / \mathrm{Ga}$ ratio of the rod-like crystals was estimated to be 20/80 from TEM-EDX measurements and the ratio was almost constant along the growth direction. A longitudinal cross-section of the GaZnNO nanocrystal prepared by FIB milling was observed using STEM. Zn/Ga elemental maps of the cross-section indicated an almost homogeneous elemental distribution in the nanocrystal, as shown in Fig. S1 (Supporting Information). Figure 3 shows the XRD pattern for a mixture of GaZnNO nanocrystals and grains prepared by ammonolysis of $\mathrm{ZnGa}_{2} \mathrm{O}_{4}$ precursor on a NiO pellet. The diffraction lines correspond to those for 
wurtzite-type GaZnNO powder. There is no trace of secondary phases such as GaNO and ZnO.

Rod-like crystals with triangular grains on the surface were never obtained by the nitridation of $\mathrm{Ga}_{2} \mathrm{O}_{3}$ and $\mathrm{ZnO}$. Nitridation of an amorphous $\mathrm{Ga}-\mathrm{Ni}$ oxide precursor formed thinner plain nanowires with smooth surfaces $[13,14]$. The characteristic sawtooth nanorod morphology appeared only for crystal growth from the $\mathrm{ZnGa}_{2} \mathrm{O}_{4}$ precursor. To investigate the growth mechanism of the GaZnNO rod-like crystals from the $\mathrm{ZnGa}_{2} \mathrm{O}_{4}$ precursor, the morphology of the nitrided product was observed by changing the nitridation time from 0 to $10 \mathrm{~h}$. For the $0 \mathrm{~h}$ nitridation, the cooling step was started just after the heating temperature reached to the setting value $\left(750^{\circ} \mathrm{C}\right)$ without a holding time. For a nitridation time of $0 \mathrm{~h}$, thinner plain nanowires with smooth surfaces appeared with large grains, as shown in Fig. 4(a). Very small amount of $\mathrm{Zn}$ below 5\% was observed in the EDX measurement of the smooth nanowires. The morphology was similar to the nitrided products obtained from a $\mathrm{Ga}_{2} \mathrm{O}_{3}$ precursor. By extending the duration time up to $5 \mathrm{~h}$, the amount of nanowires increased and small grains were precipitated on the surface of the thicker plain nanowires (Fig. 4(b)). The $Z n / G a$ ratio on the nanowires increased to about $15 / 85$. After a 
duration time of $10 \mathrm{~h}$, triangular grains covered the surface of the plain nanowire (Fig. 4(c)). The growth mechanism was speculated from the morphological changes as follows. The nanowires at the initial stage (Fig. 4(d)) were similar to the GaNO plain nanowires grown from a $\mathrm{Ga}_{2} \mathrm{O}_{3}$ precursor. Gallium oxide-rich residual may be present on the NiO pellet due to the higher vapor pressure of $\mathrm{ZnO}$ than $\mathrm{Ga}_{2} \mathrm{O}_{3}$ in $\mathrm{ZnGa}_{2} \mathrm{O}_{4}$. The $\mathrm{GaNO}$ nanowires then acted as a substrate with nucleation site for subsequent precipitations of zinc oxide deposited from the ZnO-rich atmosphere (Fig. 4(e)). At the final stage, the deposits react with the nanowire core and grow in the transverse direction, which results in the thicker rod-like nanocrystals covered in triangular grains with homogeneous $\mathrm{Zn} / \mathrm{Ga}$ distributions with $20 \%$ of $\mathrm{Zn}$ content. EDX point measurements on the both core part and triangular grain indicated almost same $\mathrm{Zn} / \mathrm{Ga}$ ratio as described in Fig. S1. Core-shell type compositional distribution was not observed in the EDX measurements on the nanocrystals. Electron diffraction (ED) patterns observed at the same positions appeared to be identical as shown in Figs. S1(d) and S1(e). The results of XRD and TEM-ED and the compositional homogeneity observed on the nanocrystal indicate the formation of GaZnNO nanorod crystals. The triangular feature of the GaZnNO grains was also 
observed in ammonolysis products of gallium zinc layered double hydroxides [23]. Triangular overgrowth along the hexagonal <001> axis suggests restructuring where GaZnNO was formed during ammonolysis through reaction of the GaNO core with the ZnO-rich atmosphere.

\section{3. $\mathrm{CL}$ characteristics}

Figure 5 shows $\mathrm{CL}$ spectra obtained from GaZnNO powder and the rod-like nanocrystals. Both spectra exhibit broad emissions centered at around $2.1 \mathrm{eV}$. The emission energy of the rod-like nanocrystals does not change in the growth direction, which implies a homogeneous chemical composition. Band edge emissions of $\mathrm{GaN}(3.4 \mathrm{eV})$ and $\mathrm{ZnO}(3.2 \mathrm{eV})$ binary compounds do not appear in the spectra. Many studies on $\mathrm{Ga}_{1-x} \mathrm{Zn}_{x} \mathrm{~N}_{1-x} \mathrm{O}_{x}$ solid solution photocatalysts have investigated the relationship between the band gap energy and the chemical compositions [18, 19, 21-23]. Among the previous reports, the smallest band gap energy of $2.13 \mathrm{eV}$ was achieved for a Zn-rich Ga oxynitride with $\mathrm{Zn} / \mathrm{Ga}=0.9 / 0.1$ [19]. The $\mathrm{Ga}_{1-\mathrm{x}} \mathrm{Zn}_{\mathrm{x}} \mathrm{N}_{1-\mathrm{x}} \mathrm{O}_{\mathrm{x}}$ solid solution with $\mathrm{x} \approx 0.20$ exhibited a band gap energy of around $2.6 \mathrm{eV}[18,23]$. The band gap energy was smaller than those for $\mathrm{GaN}$ and $\mathrm{ZnO}$, and has been interpreted as an 
enhanced valence-band maximum caused by a repulsion of $N-2 p$ and $\mathrm{Zn}-3 \mathrm{~d}$ orbitals [17, 32]. The emission at $2.1 \mathrm{eV}$ observed in this study for both GaZnNO powder and the rod-like nanocrystals with $\mathrm{Zn} / \mathrm{Ga}$ ratios of $0.23 / 0.75$ and $0.20 / 0.80$, respectively, is much lower in energy than the band gap for $\left(\mathrm{Ga}_{1-x} \mathrm{Zn}_{\mathrm{x}}\right)\left(\mathrm{N}_{1-\mathrm{x}} \mathrm{O}_{\mathrm{x}}\right)$ solid solutions with similar $\mathrm{Zn} / \mathrm{Ga}$ compositional ratios. In this study, the oxynitride powder has a chemical composition of $\mathrm{Ga}_{0.75} \mathrm{Zn}_{0.23} \mathrm{~N}_{0.71} \mathrm{O}_{0.29}$. This powder suggests the wurtzite structure and thus a small amount of cation vacancies should be present in the structure. The effects of cation vacancies, $V_{G a}$, and oxide ion substitution, $\mathrm{O}_{\mathrm{N}}$, on the emission spectra have been reported for wurtzite type GaN $[9,10]$. The yellow emission at $2.2 \mathrm{eV}$ has been assigned to a correlation of $V_{G a}$ and $O_{N}$ [9]. $\mathrm{Zn}$ - doped $\mathrm{GaN}$ has also been reported to exhibit blue emission due to a $\mathrm{Zn}$ accepter level. In this study, both $\mathrm{O}_{\mathrm{N}}$ and $\mathrm{V}_{\mathrm{Ga}}$ may produce the slightly lower emission around $2.1 \mathrm{eV}$ due to a coexistence of $\mathrm{Zn}_{\mathrm{Ga}}$ which forms an accepter-like level. The anion composition of the rod-like nanocrystals was not estimated due to the difficulty in measurement of a precise O/N ratio from TEM-EDX spectra. Although the entire chemical composition of the rod-like nanocrystals was not clear, the similar $C L$ emission spectra (peak energy and broad emission) for the GaZnNO nanocrystals and powder imply the 
presence of cation vacancies in the nanocrystals. Cation vacancies have also been reported in spinel-type gallium and aluminum oxynitrides [33,34]. Wurtzite-type Al-O-N polytypoids also contains several amount of cation vacancies [34]. Anion site splitting model in wurtzite-type $\mathrm{Ga}_{1-x} \mathrm{Zn}_{x} \mathrm{~N}_{1-x} \mathrm{O}_{x}(x=$ 0.115 ) has been suggested by a structural refinement using a synchrotron X-ray diffraction data [32]. The anion site splitting implies a formation of stacking disorder in the hexagonal wurtzite and cubic zinc-blende lattices indicating a cation deficient layer in the structure as observed in GaNO nanowires [14]. Further detailed investigation is required on the precise estimation of the chemical composition and cation vacancies in relation to the optical property of the nanocrystals.

\section{Conclusion}

Gallium zinc oxynitride powder containing a small amount of cation vacancies was obtained by nitridation of $\mathrm{ZnGa}_{2} \mathrm{O}_{4}$ at $750{ }^{\circ} \mathrm{C}$ under $\mathrm{NH}_{3}$ flow. The crystallinity was improved with respect to that observed for gallium oxynitride. Rod-like nanocrystals of gallium zinc oxynitride formed on a NiO catalyst pellet had a sawtooth-like appearance with triangular grains on the nanorod surfaces. 
The nanocrystals with a $\mathrm{Ga} / \mathrm{Zn}$ ratio of $0.80 / 0.20$ exhibited a broad $\mathrm{CL}$ emission at $2.1 \mathrm{eV}$, similar to the gallium zinc oxynitride powder. Cation vacancies may be present in the oxynitride nanocrystals, as observed in the powder products, which may lead to the smaller CL emission energy through a cooperative effect between $\mathrm{Zn}$ substituted on $\mathrm{Ga}$ sites and oxide ions in the wurtzite lattice.

\section{Acknowledgements}

This research was partly supported by a Grant-in-Aid for Young Scientists (B) (No. 24750196) from the Japan Society of the Promotion of Science (JSPS). A. K. and Y. M. appreciate Dr. Kentaro Watanabe at the National Institute for Materials Science (NIMS) of Japan for his valuable support with the $C L$ measurements. 


\section{References}

[1] A.M.S. ElAhl, M. He, P. Zhou, G.L. Harris, L. Salamanca-Riba, F. Felt, H.C.

Shaw, A. Sharma, M. Jah, D. Lakins, T. Steiner, S.N. Mohammad, Systematic study of effects of growth conditions on the (nano-, meso-, micro) size and (one-, two-, three-dimensional) shape of GaN single crystals grown by a direct reaction of Ga with ammonia, J. Appl. Phys. 94 (2003) 7749-4456

[2] Y. Huang, X. Duan, Y. Cui, L.J. Lauhon, K.-H. Kim, C.M. Lieber, Logic gates and computation from assembled nanowire, Sci. 294 (2001) 1313-1317

[3] J.C. Johnson, H.-J. Choi, K.P. Knutsen, R.D. Schaller, P. Yang, R.J. Saykally, Single gallium nitride nanowire lasers, Nat. Mater. 1 (2002) 106-110

[4] Y. Xia, P. Yang, Y. Sun, Y. Wu, B. Mayers, B. Gates, Y. Yin, F. Kim, H. Yan, One-dimensional nanostructures: synthesis, characterization, and applications, Adv. Mater. 15 (2003) 353-389

[5] S.P. Lau, L. Huang, S.F. Yu, H. Yang, J.K. Yoo, S.J. An, G.-C. Yi, Enhanced secondary electron emission from group III nitride/ZnO coaxial nanorod heterostructures, Small 2 (2006) 736-740

[6] A. Motayed, M. Vaudin, A.V. Davydov, J. Melngailis, M. He, S.N. Mohammad, Diameter dependent transport properties of gallium nitride nanowire field effect 
transistors, Appl. Phys. Lett. 90 (2007) 043104/1-3

[7] J.K. Sheu, G.C. Chi, The doping process and dopant characteristics of GaN,

J. Phys.: Condens. Matter 14 (2002) R657-R702

[8] J.B. Webb, H. Tang, S. Rolfe, J.A. Bardwell, Semi-insulating C-doped GaN and high-mobility AIGaN/GaN heterostructures grown by ammonia molecular beam epitaxy, Appl. Phys. Lett. 75 (1999) 953-955

[9] M.A. Reshchikova, H. Morkoc, Luminescence properties of defects in GaN, J. Appl. Phys. 97 (2005) 061301/1-95

[10] M. Toth, K. Fleischer, M.R. Phillips, Direct experimental evidence for the role of oxygen in the luminescent properties of GaN, Phys. Rev. B 59 (1999) $1575-1578$

[11] M. Kang, J.-S. Lee, S.-K. Sim, H. Kim, B. Min, K. Cho, G.-T. Kim, M.-Y. Sung, S. KIM and H.S. Han, Photocurrent and photoluminescence characteristics of networked GaN nanowires, Jpn. J. Appl. Phys. 43 (2004) 6868-6872

[12] S. Kikkawa, K. Nagasaka, T. Takeda, M. Bailey, T. Sakurai, Y. Miyamoto, Preparation and lithium doping of gallium oxynitride by ammonia nitridation via a citrate precursor route, J. Solid State Chem. 180 (2007) 1984-1989

[13] A. Miyaake, Y. Masubuchi, T. Takeda, T. Motohashi, S. Kikkawa, Preparation 
of gallium oxynitride powder and its nanofibers by the nitridation of a gallium oxide precursor dopoed with nickel or cobalt obtained via the citrate route, Dalton Trans. 39 (2010) 6106-6111

[14] Y. Masubuchi, R. Yamaoka, T. Tohei, T. Mizoguchi, Y. Ikuhara, S. Kikkawa, Nanowire of hexagonal gallium oxynitride: Direct observation of its stacking disorder and its long nanowire growth, J. Eur. Ceram. Soc. 32 (2012) 1989-1993 [15] Y. Masubuchi, R. Yamaoka, T. Motohashi, K. Kirihara, W. Lee, K. Watanabe, T. Sekiguchi, S. Kikkawa, Crystal growth and characterization of gallium oxynitride nanowires grown on seed crystals, J. Crys. Growth 337 (2011) 87-92

[16] A. Miyaake, Y. Masubuchi, T. Takeda, S. Kikkawa, Indium and gallium oxynitrides prepared in the presence of $\mathrm{Zn}^{2+}$ by ammonolysis of the oxide precursors obtained via the citrate route, Mater. Res. Bull. 45 (2010) 505-508 [17] K. Maeda, T. Takata, M. Hara, N. Saito, Y. Inoue, H. Kobayashi, K. Domen, GaN:ZnO solid solution as a photocatalyst for visible-light-driven overall water splitting, J. Am. Chem. Soc. 127 (2005) 8286-8287

[18] K. Maeda, K. Teramura, T. Takata, M. Hara, N. Saito, K. Toda, T. Inoue, H. Kobayashi, K. Domen, Overall water splitting on $\left(\mathrm{Ga}_{1-x} \mathrm{Z} \mathrm{n}_{x}\right)\left(\mathrm{N}_{1-\mathrm{x}} \mathrm{O}_{\mathrm{x}}\right)$ solid solution photocatalyst: relationship between physical properties and photocatalytic 
activity, J. Phys. Chem. B 109 (2005) 20504-20510

[19] Y. Li, L. Zhu, Y. Yang, H. Song, Z. Lou, Y. Guo, Z. Ye, A full compositional range for a $\left(\mathrm{Ga}_{1-\mathrm{x}} \mathrm{Zn}_{\mathrm{x}}\right)\left(\mathrm{N}_{1-\mathrm{x}} \mathrm{O}_{\mathrm{x}}\right)$ nanostructure: high efficiency for overall water splitting and optical properties, Small 11 (2015) 871-876

[20] M.J. Ward, W.-Q. Han, T.-K. Sham, Nitridation temperature effects on electronic and chemical properties of $\left(\mathrm{Ga}_{1-\mathrm{x}} \mathrm{Zn}_{\mathrm{x}}\right)\left(\mathrm{N}_{1-\mathrm{x}} \mathrm{O}_{\mathrm{x}}\right)$ solid solution nanocrystals, J. Phys. Chem. C 117 (2013) 20332-20342

[21] B. Adeli, F. Taghipour, A review of synthesis techniques for gallium-zinc oxynitride solar-activated photocatalyst for water splitting, J. Solid State Technol. 2 (2013) Q118-Q126

[22] B. Adeli, F. Taghipour, Facile synthesis of highly efficient nano-structured gallium zinc oxynitride solid solution photocatalyst for visible-light overall water splitting, Appl. Catal. A: Gen. 521 (2016) 250-258

[23] D.P. Chen, S.E. Skrabalak, Synthesis of $\left(\mathrm{Ga}_{1-\mathrm{x}} \mathrm{Zn}_{\mathrm{x}}\right)\left(\mathrm{N}_{1-\mathrm{x}} \mathrm{O}_{\mathrm{x}}\right)$ with enhanced visible-light absorption and reduced defects by suppressing $\mathrm{Zn}$ volatilization, Inorg. Chem. 55 (2016) 3822-3828

[24] W.-Q. Han, Y. Zhang, C.-Y. Nam, C. T. Black, E.E. Mendez, Growth and electronic properties of GaN/ZnO solid solution nanowires, Appl. Phys. Lett. 97 
(2010) 83108/1-3

[25] M. Zhong, Y. Ma, P. Oleynikov, K. Domen, J.J. Delaunay, A conductive ZnO-ZnGaON nanowire-array-on-a-film photoanode for stable and efficient sunlight water splitting, Energy Environ. Sci. 7 (2014) 1693-1699

[26] S. G. Ebbinghaus, H. P. Abicht, R. Dronskowski, T. Muller, A. Reller, A. Weidenkaff, Perovskite-related oxynitrides - Recent developments in synthesis, characterisation and investigations of physical properties, Prog. Solid State Chem. 37 (2009) 173-205

[27] G. Kamler, J. Zachara, S. Podsiadlo, L. Adamowicz, W. Gebicki, Bulk GaN single-crytals growth, J. Crys. Growth 212 (2000) 39-48

[28] H. Yamane, M. Shimada, F.J. DiSalvo, Growth of zinc-blend-type structure GaN from a Na-Ga melt, Mater. Lett. 42 (2000) 66-70

[29] R. D. Shannon, Revised effective ionic radii and systematic studies of interatomic distances in halides and chalcogenides, Acta Crystallogr. A A32 (1976) 751-767

[30] S. Kikkawa, K. Nagasaka, T. Takeda, M. Bailey, T. Sakurai, Y. Miyamoto, Preparation and lithium doping of gallium oxynitride by ammonia nitridation via a citrate precursor route, J. Solid State Chem. 180 (2007) 1984-1989 
[31] S. C. Abrahams, J. L. Bernstein, Remeasurement of the structure of hexagonal ZnO, Acta Cryst. B25 (1969) 1233-1236

[32] M. Yashima, H. Yamada, K. Maeda, K. Domen, Experimental visualization of covalent bonds and structural disorder in a gallium zinc oxynitride photocatalyst $\left(\mathrm{Ga}_{1-x} \mathrm{Zn}_{\mathrm{x}}\right)\left(\mathrm{N}_{1-\mathrm{x}} \mathrm{O}_{\mathrm{x}}\right)$ : origin of visible light absorption, Chem. Commun. 46 (2010) $2379-2381$

[33] H. Huppertz, S. A. Hering, C. E. Zvoriste, S. Lauterbach, O. Oeckler, R. Riedel, I. Kinski, High-pressure synthesis, electron energy-loss spectroscopy investigations, and single crystal structure determination of a spinel-type gallium oxonitride $\mathrm{Ga}_{2.79} \square_{0.21}\left(\mathrm{O}_{3.05} \mathrm{~N}_{0.76} \square_{0.19}\right)$, Chem. Mater. 21 (2009) 2101-2107

[34] N. D. Corbin, Aluminum oxynitride spinel: A review, J. Eur. Ceram. Soc. 5 (1989) $143-154$ 
Table 1 Estimated chemical compositions for the nitrided products

\begin{tabular}{llcccc}
\hline \multirow{2}{*}{ Samples } & \multirow{2}{*}{ Compositions } & \multicolumn{2}{c}{ XRF } & \multicolumn{2}{c}{ O/N analysis } \\
\cline { 3 - 6 } & & $\mathrm{Ga} / \mathrm{at} \%$ & $\mathrm{Zn} / \mathrm{at} \%$ & $\mathrm{~N} / \mathrm{wt} \%$ & $\mathrm{O} / \mathrm{wt} \%$ \\
\hline GaNO & $\left(\mathrm{Ga}_{0.92(1)} \square_{0.08(1)}\right)\left(\mathrm{N}_{0.767(5)} \mathrm{O}_{0.233(5)}\right)$ & 100 & - & $13.6(2)$ & $4.7(2)$ \\
GaZnNO & $\left(\mathrm{Ga}_{0.750(3)} \mathrm{Zn}_{0.22(2)} \square_{0.024(5)}\right)\left(\mathrm{N}_{0.705(2)} \mathrm{O}_{0.295(2)}\right)$ & $76.8(1)$ & $23.2(1)$ & $11.5(1)$ & $5.5(1)$ \\
\hline
\end{tabular}

Symbol $\square$ indicates a cation vacancy. 
Figure captions

Figure 1. XRD patterns for the nitrided (a) GaZnNO and (b) GaNO powders prepared without NiO. Calculated XRD patterns for (c) zinc-blende GaN and (d) wurtzite type GaN $[27,28]$ are also presented.

Figure 2. SEM images of the nitrided products obtained from (a) $\mathrm{Ga}_{2} \mathrm{O}_{3}$ and (b) $\mathrm{ZnGa}_{2} \mathrm{O}_{4}$ precursors on a $\mathrm{NiO}$ pellet. (c) A magnified image of a rod-like nanocrystal shown in (b).

Figure 3. XRD pattern of GaZnNO nanocrystals prepared on a NiO pellet. The inset shows a magnified image of the higher angle region. Vertical bars indicate the Bragg reflections for GaNO (upper) with $\mathrm{a}=0.3189 \mathrm{~nm}$ and $\mathrm{c}=0.5181 \mathrm{~nm}$ [30], GaZnNO (middle) with $\mathrm{a}=0.3204 \mathrm{~nm}$ and $\mathrm{c}=0.5195 \mathrm{~nm}$, and $\mathrm{ZnO}$ (lower) with $\mathrm{a}=0.3249 \mathrm{~nm}$ and $\mathrm{c}=0.5206 \mathrm{~nm}$ [31]. Lattice parameters for the wurtzite-type GaZnNO were estimated for the powder sample obtained in this study.

Figure 4. SEM images of the products obtained by nitridation of $\mathrm{ZnGa}_{2} \mathrm{O}_{4}$ at 
$750^{\circ} \mathrm{C}$ for (a) $0 \mathrm{~h}$, (b) $5 \mathrm{~h}$, and (c) $10 \mathrm{~h}$. (d-f) Growth mechanism of the rod-like GaZnNO nanocrystals.

Figure 5. CL spectra for GaZnNO (a) powder and (b) rod-like nanocrystal. The inset shows an SEM image of a rod-like nanocrystal, where the numbers correspond to the positions of CL measurements. 
Figure 1

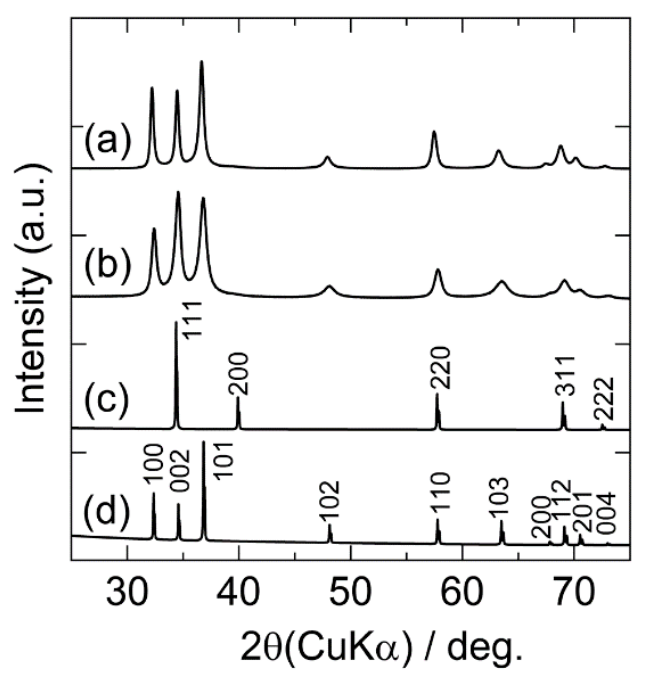

Figure 1. XRD patterns for the nitrided (a) GaZnNO and (b) GaNO powders prepared without NiO. Calculated XRD patterns for (c) zinc-blende GaN and (d) wurtzite type GaN $[27,28]$ are also presented. 
Figure 2
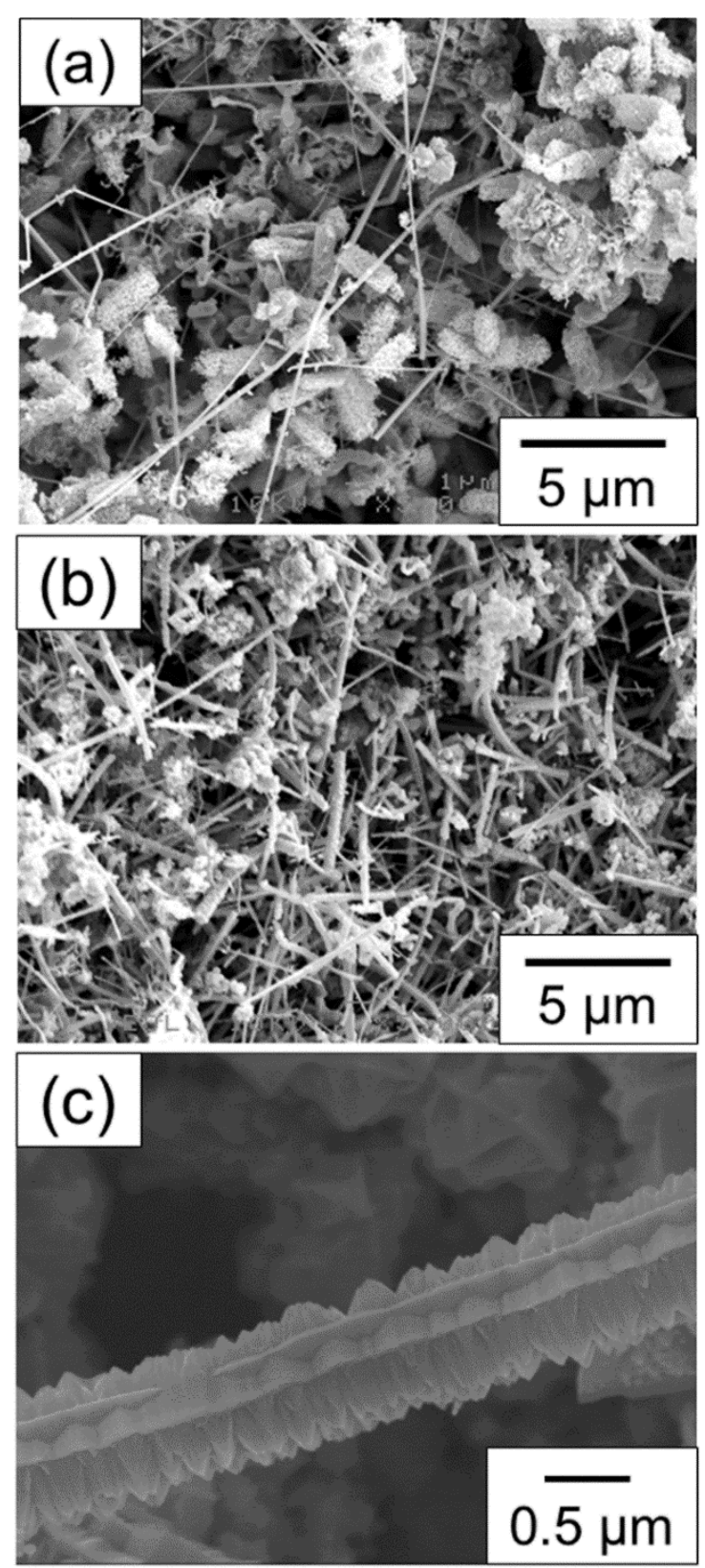

Figure 2. SEM images of the nitrided products obtained from (a) $\mathrm{Ga}_{2} \mathrm{O}_{3}$ and (b) $\mathrm{ZnGa}_{2} \mathrm{O}_{4}$ precursors on a $\mathrm{NiO}$ pellet. (c) A magnified image of a rod-like nanocrystal shown in (b). 
Figure 3

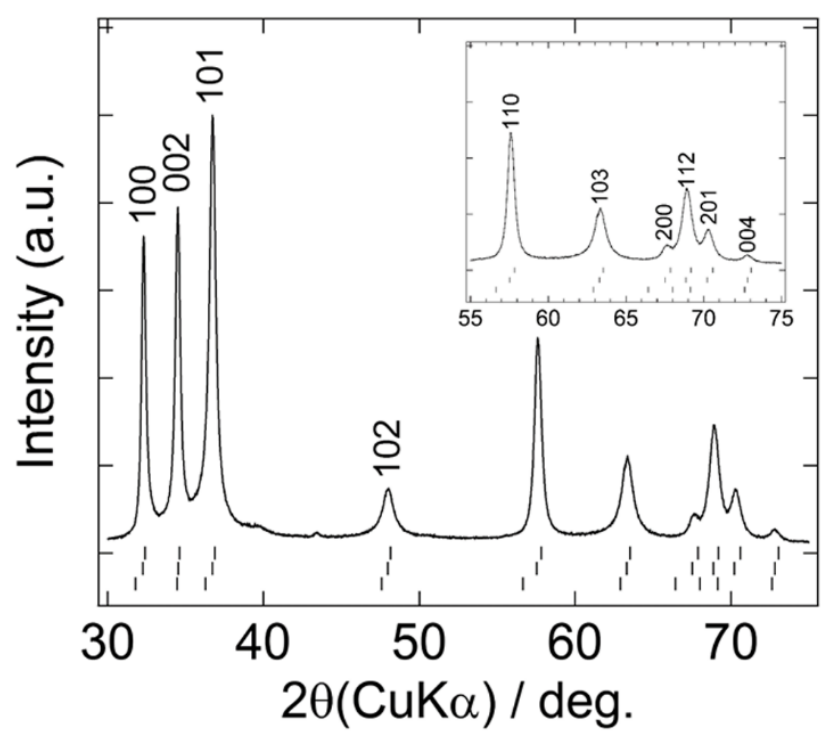

Figure 3. XRD pattern of GaZnNO nanocrystals prepared on a NiO pellet. The inset shows a magnified image of the higher angle region. Vertical bars indicate the Bragg reflections for GaNO (upper) with $\mathrm{a}=0.3189 \mathrm{~nm}$ and $\mathrm{c}=0.5181 \mathrm{~nm}$ [30], GaZnNO (middle) with $\mathrm{a}=0.3204 \mathrm{~nm}$ and $\mathrm{c}=0.5195 \mathrm{~nm}$, and $\mathrm{ZnO}$ (lower) with $\mathrm{a}=0.3249 \mathrm{~nm}$ and $\mathrm{c}=0.5206 \mathrm{~nm}$ [31]. Lattice parameters for the wurtzite-type GaZnNO were estimated for the powder sample obtained in this study. 
Figure 4

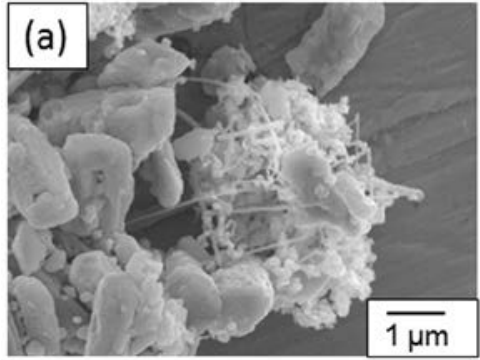

(d) Growth of $\mathrm{Ga}(\mathrm{N}, \mathrm{O})$ nanowire

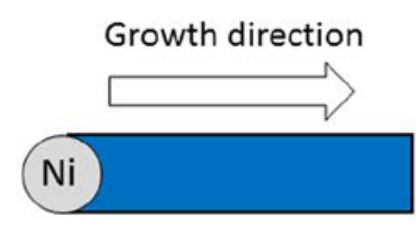

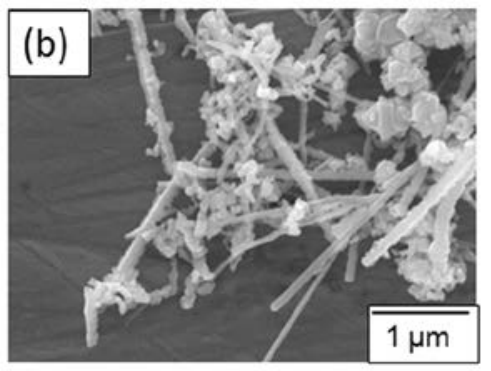

(e) ZnO precipitation on the NW

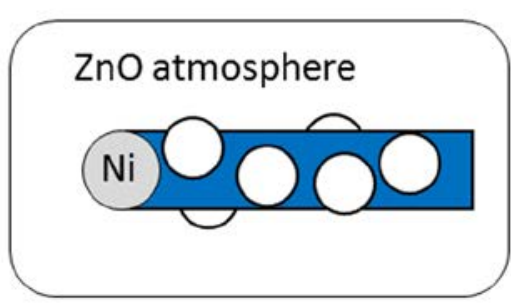

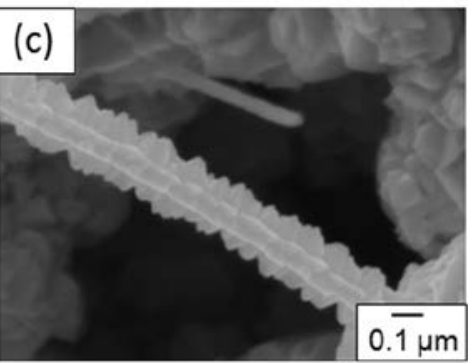

(f) Growth in transverse direction, to form $(\mathrm{Ga}, \mathrm{Zn})(\mathrm{O}, \mathrm{N})$

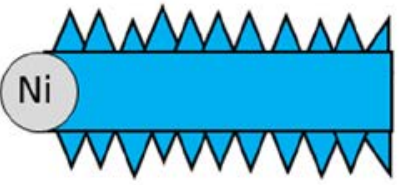

Figure 4. SEM images of the products obtained by nitridation of $\mathrm{ZnGa}_{2} \mathrm{O}_{4}$ at $750{ }^{\circ} \mathrm{C}$ for (a) $0 \mathrm{~h},(\mathrm{~b}) 5 \mathrm{~h}$, and (c) $10 \mathrm{~h}$. (d-f) Growth mechanism of the rod-like GaZnNO nanocrystals. 
Figure 5

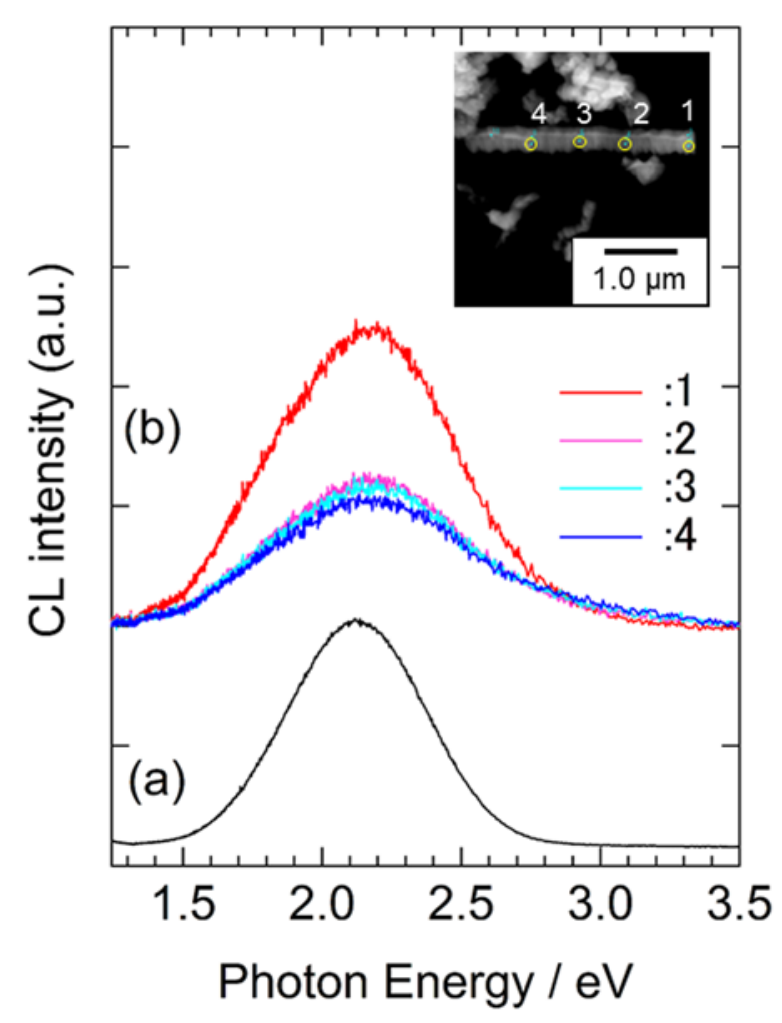

Figure 5. CL spectra for GaZnNO (a) powder and (b) rod-like nanocrystal. The inset shows an SEM image of a rod-like nanocrystal, where the numbers correspond to the positions of CL measurements. 